http://dx.doi.org/10.22402/j.rdipycs.unam.1.2.2015.24.263-276

\title{
RELACIÓN ENTRE NIVEL DE ANSIEDAD Y FUNCIONAMIENTO FAMILIAR
}

\author{
Lourdes Remigio-Mondragón y Angélica Pérez-Arredondo \\ INSTITUTO FAMILIAR SISTÉMICO ACT \\ MÉXICO
}

\begin{abstract}
RESUMEN
El presente trabajo tiene como objetivo describir la relación existente entre la funcionalidad de la familia y las causas de la ansiedad en algunos de sus miembros. Se encontró que la madre ha presentado ansiedad la mayor parte de su vida de casada. Acude la familia a terapia solicitando el servicio psicológico para el hijo mayor de 20 años que presenta crisis de ansiedad y preocupación por su padre cuando éste se encuentra fuera de casa. Se trabajó con intervenciones principalmente bajo el modelo centrado en soluciones y, como herramienta complementaria, técnicas de hipnosis ericksoniana. Desde la segunda sesión fueron reportando cambios significativos en las interacciones familiares y en el autocontrol de la ansiedad que la madre presentaba y que su hijo había aprendido, ahora ella interactuaba de forma asertiva con su esposo e hijos y, en consecuencia, el hijo ya no presentaba síntomas de ansiedad.
\end{abstract}

Palabras Clave:

Ansiedad, familia, modelo centrado en soluciones, hipnosis ericksoniana.

\section{RELATIONSHIP BETWEEN THE LEVEL OF ANXIETY AND FAMILY FUNCTIONING}

\begin{abstract}
This paper aims to describe the relationship between family functioning and causes anxiety in some of its members. It was found that the mother has presented anxiety most of her married life. Family comes to seeking psychological therapy service for the eldest son of 20 years who has panic attacks and concern for his dad when he is away from home. We worked with interventions mainly in the model focused on solutions and complementary techniques as Ericksonian hypnosis tool. Since the second session were reporting significant changes in family interactions and self-anxiety that the mother had and that his son had learned, now she interacted assertively with her husband and children, and consequently, the son not had symptoms of anxiety.
\end{abstract}

Keywords:

Anxiety, family, solution-focused model, Ericksonian hypnosis.

Bitácora del Artículo:

| Recibido: 03 de octubre de 2015 | Aceptado: 06 mayo de 2016 | Publicado en línea: 15 de diciembre de 2015 | 


\title{
RELACIÓN ENTRE NIVEL DE ANSIEDAD Y FUNCIONAMIENTO FAMILIAR
}

\author{
Lourdes Remigio-Mondragón y Angélica Pérez-Arredondo
}

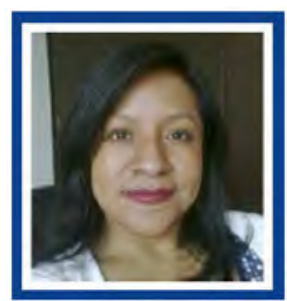

Lourdes Remigio-Mondragón Instituto Familiar Sistémico ACT

Correo: lourdesremm@gmail.com

Licenciada en Psicología por la Universidad del Valle de México y Maestra en Hipnosis y Terapia Familiar Breve Sistémica por la Universidad José Martí de Latinoamérica e INFASI ACT. Diplomada en Terapia Familiar Breve Sistémica y en Hipnosis Clínica Ericksoniana, por la Universidad Autónoma de Nuevo León.

Ver más...

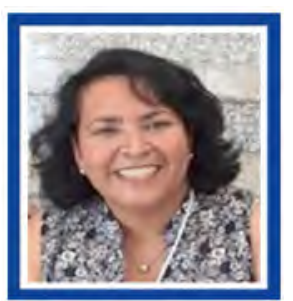

Angélica Pérez-Arredondo

Instituto Familiar Sistémico ACT

Correo: para_angelica@hotmail.com

Maestra en Psicología Educativa, de la Facultad de Psicología, UNAM y egresada de la licenciatura en Psicología de la ENEP Iztacala-UNAM. Diplomada en Filosofía; en Terapia Familiar Breve Sistémica, y en Hipnosis Clínica Ericksoniana.

Ver más...

\section{CONTRIBUCIÓN DE LOS AUtORAS}

La primera autora realizó el trabajo terapéutico y la segunda lo supervisó. Ambas redactaron el artículo.

\section{AgRADECIMIENTOS}

Las autoras del presente trabajo agradecen la supervisión del caso, guiada por la Mtra. Alejandra Andrade, profesora de la Maestría en Hipnosis y Terapia Familiar Breve Sistémica y Directora Administrativa de INFASI ACT.

\section{DATOS DE FILIACIÓN DE LAS AUTORAS}

Primera autora egresada de la Maestría en Hipnosis y Terapia Familiar Breve Sistémica, INFASI ACT. Segunda autora profesora del Instituto Familiar Sistémico

Copyright: (c) 2015 Remigio-Mondragón, L. \& Pérez-Arredondo, A.

Este es un artículo de acceso abierto distribuido bajo los términos de la licencia Creative Commons Reconocimiento-NoComercial 4.0 Internacional, por lo que su contenido gráfico y escrito se puede compartir, copiar y redistribuir total o parcialmente sin necesidad de permiso expreso de sus autoras con la única condición de que no se puede usar con fines directamente comerciales y los términos legales de cualquier trabajo derivado deben ser los mismos que se expresan en la presente declaración. La única condición es que se cite la fuente con referencia a la Revista Digital Internacional de Psicología y Ciencia Social y a sus autoras. 


\section{TABLA DE CONTENIDO}

INTRODUCCIÓN

266

La intervención terapéutica, 266

MÉTODO

268

Identificación del caso, 268

Análisis del motivo de la intervención, 268

Antecedentes del problema, 268

Descripción y análisis del problema, 268

Plan de InTERVEnción

Descripción del plan de intervención, 269

Establecimiento de los objetivos y las metas del plan de intervención, 269

Técnicas de evaluación del plan de intervención, 269

Aplicación del plan de intervención, 270

PRESENTACIÓN DE RESULTADOS

Análisis de los cambios que produjo el plan de intervención, 272

CONCLUSIONES

REFERENCIAS

Meta-Analisis del Artículo

Dimensión Cuantitativa, 278

Dimensión Cualitativa, 280

Historia del Proceso Editorial

283 


\section{INTRODUCCIÓN}

$\mathrm{L}$

os datos sobre la ansiedad en nuestro país han sido alarmantes desde hace más de una década, la información reportada por la Encuesta Nacional de Epidemiología Psiquiátrica (2003; citado en Fuentes, 2013) la ubica como uno de los trastornos más frecuentes en nuestro país, con una prevalencia de $14.3 \%$ alguna vez en la vida de las personas, seguidos por los trastornos por uso de sustancias $(9.2 \%)$ y los trastornos afectivos (9.1\%).

Para Goldman (2001; como se citó en Zavala, Ríos, García \& Rodríguez, 2009), la ansiedad es considerada como un estado emocional que frecuentemente se acompaña de síntomas fisiológicos que pueden conducir a la fatiga o incluso al agotamiento. Aunque más preciso es Isaacs (2000), quien la define como una respuesta subjetiva al estrés, como una sensación de aprensión, incertidumbre o temor, a consecuencia de una amenaza real o percibida cuyo origen se desconoce o no es reconocido.

Según Yalóm (como se citó en De Castro \& García, 2011), la ansiedad "surge a partir de la conciencia que se tiene de estos supuestos básicos, en el ser humano siempre habrá en algún grado la posibilidad de que sus valores se destruyan, que no los pueda seguir afirmando o que fracase en algún proyecto que sea valioso para sí, lo que es un generador de ansiedad en la persona" (p. 45). Y así como lo menciona Goldman (2001), la intensidad de la ansiedad puede ir variando en grados desde inquietudes menores hasta temblores notables e incluso crisis de angustia completa, con síntomas como preocupación excesiva, tensión muscular, alteraciones del sueño, sentimiento de agitación, fatiga, dificultad para concentrarse, la persona encuentra difícil controlar su preocupación, entre otros.

Es a la ansiedad y sus diversas manifestaciones a la que se puede anteponer como las causas de reprobación escolar (Rosário, Núñez, Salgado, González-Pineda, Valle, Joly \& Bernardo, 2008), del estrés y depresión (Méndez, Giraldoscar, Aguirre-Acevedo \& Lopera, 2010), de la salud en general (Soria, Ávila \& Rodríguez, 2014), la satisfacción familiar (Salazar, Veytia, Márquez \& Huitrón, 2013) o bien de la influencia del clima fami- liar y estrés del padre de familia en la salud mental de los niños (Quintana \& Sotil, 2014), entre otros.

En un viejo estudio realizado por Alchaer, Bahsas, Hernández y Salinas (1994) sobre el funcionamiento familiar y su relación con el estrés y la ansiedad, los resultados mostraron que el nivel de funcionamiento puede afectar a aquellas influencias familiares controlables por el hombre, de manera tal que éstas se reflejen positiva o negativamente en la salud de sus individuos. Además, el funcionamiento familiar puede verse alterado por cualquiera de las otras variables que configuran el ambiente social. Por ejemplo, en este estudio se observó que los adolescentes, los divorciados, los del estrato socioeconómico laboral obrero, los obesos, los alcohólicos y los que pasaban por una enfermedad psicosomática, presentaban mayores niveles de disfunción familiar que otros grupos estudiados. La desventaja de este tipo de investigaciones es que, enfocados en la predictibilidad de instrumentos como el APGAR, omitieron la consideración de la capacidad de respuesta del individuo para lograr el equilibrio emocional frente a dichas agresiones. Otro dato relevante de ese trabajo fue la inconsistencia entre lo que se reporta en una escala y lo observado, ya que a pesar de que las causas de disfunción familiar más frecuente fueron la insatisfacción de la ayuda recibida por la familia y la manera como se comparte el tiempo, el espacio, el dinero y los recursos existentes, los participantes con disfunción familiar en casi la mitad de los casos manifestaron en la encuesta de APGAR Familiar II, que tenían buenas relaciones con todos los miembros de su familia.

Retomando estos resultados, para el presente trabajo nos basamos en la hipótesis de que la relación funcional de la familia se veía influida por la ansiedad que presentaba la madre, y que posiblemente era producto de un conflicto encubierto en el holón conyugal y el síntoma era exteriorizado por los hijos. En terapia se ha encontrado que cuando la ansiedad (a veces interpretada o percibida como temor, inseguridad, celos, etc.) les sucede a los padres, tienden a sobreproteger a sus hijos, ya que ellos viven en una constante zozobra por temor a perder a sus padres o que algo malo les pudiera pasar, y viceversa.

\section{La intervención terapéutica}

Desde y en el sistema. Haley (1991) consideraba que lo importante en el trabajo terapéutico es, cuántas relaciones están en la mente del terapeuta porque de ello se de- 
riva el camino a seguir. Desde la perspectiva que se parte para el presente caso, Charles (2008) supone que el terapeuta sistémico ve al individuo en su contexto, y que el sistema que se crea y se conjunte para la solución del problema es diferente del sistema en el cual se originó el problema, ya que este nuevo sistema se conforma por todos y todo aquello que le da significado para quien tiene los problemas, y que además quiere colaborar para solucionarlo. Por tanto, en la definición del objetivo terapéutico queda implícito que habrá que comenzar por definir quién es cliente (el que está dispuesto a pagar, el que hace la primera llamada y que está dispuesto a hacer un cambio, regularmente al que le duele más, y en este momento está preocupado) y quién paciente, para así seguir identificando las interacciones que se den, así como el tipo de éstas. Se trata aquí de que la ansiedad ha sido mantenida por un sistema que parecía óptimo hasta que ya no resultó serlo para toda la familia.

La adherencia en terapia. La terapia cubre varias fases, ya que se trabaja con los miembros del sistema (estén o no presentes en el consultorio), lo que interesa es saber el nivel en el que se debe abordar la problemática, comenzando por lo obvio para los consultantes hasta deshacer los conflictos disfrazados. Puede ser que a terapia haya llegado la familia completa, pero conforme se va analizando la problemática, ésta resulte ser mantenida sólo por algunos de los miembros. Coincidiendo con el presente caso y lo señalado por Charles, ante los conflictos encubiertos por la pareja, lo primero que Ilega a terapia es trabajar con su rol de padres para un problema prioritario de un hijo, ya que la terapia de pareja será en una segunda o tercera fase del trabajo; es decir, pudiera ser que la conducta de los niños y adolescentes de alguna manera es una metáfora de la conducta de algunos de los padres, o de la relación de los padres. Será hasta la segunda etapa en la que se pueda entrar a los problemas de comunicación y conflictos de relación conyugal.

Con enfoque centrado en soluciones. Como lo señala Moreno (2013), es difícil llegar a darnos cuenta de que, tras varios intentos por solucionar una situación, ésta llega a complicarse tanto que se convierte en un problema. El aspecto que subraya De Shazer (como se citó en Moreno, 2013) es que, mientras esto sucede, la persona percibe cada vez más su problema y su incapacidad de resolverlo, pasando por alto aquellas ocasiones en las que el problema no se da, o se da con menor intensidad o duración, impidiendo entonces detectar posibles variaciones y excepciones.

Por ello es que, apoyándonos en el modelo de terapia sistémica centrado en soluciones, el trabajo para la ansiedad tiene un impacto diferente a las estrategias convencionales de autocontrol. En este sentido, el objetivo de la terapia es ayudar al consultante a encontrar un modo en que pueda percibir las excepciones como "una diferencia que marca una diferencia" y aumentarlas hasta que el problema deje de ser tal. Este modelo se centra en las posibles soluciones, conduciendo a la persona que piense qué estaría haciendo diferente si no tuviera ese problema, que se imagine sin su problema, y junto con la ayuda del terapeuta se co-crea una realidad diferente ante su problema, de menos que sea manejable; parte del enfoque es que la persona vaya realizando pequeños cambios, de estos mismos que se reportan en cada sesión el terapeuta los celebra, así mismo por medio de las preguntas preposicionales se da por hecho que los cambios ya están sucediendo, cabe mencionar que una de las tareas que se formula desde la primera sesión se les pregunta a los clientes, qué cosas positivas quiere que continúe ocurriendo. (De Shazer, 1985; como se citó en O'Hanlon \& Weiner-Davis, 2010)

En el transcurso de las sesiones cuando el paciente reporta los cambios logrados estos se profundizan de tal modo que piense minuciosamente quién de la familia se dio cuenta que está logrando, en este caso, controlar la ansiedad y qué es diferente ahora, en ésta misma línea se le pide al paciente que suponga justamente que por la noche ocurre un milagro, a la mañana siguiente el problema está resuelto, quién será el primero en darse cuenta y qué cosas han cambiado.

En forma resumida, el modelo centrado en soluciones se enfoca estratégicamente en intervenciones de comunicación terapéutica como: utilización cuidadosamente del lenguaje, trabajar con las respuestas que sus miembros vayan dando y seguir preguntando para lograr que estas respuestas resulten útiles; además, será necesario gestionar sus respuestas para generar nueva información sobre los avances ya producidos, las expectativas de futuro y los pasos siguientes a dar (Beyebach, 2013). Todo ello a través de iluminar, ampliar, anclar las soluciones o atribuir el control, promover activamente la cooperación con la familia, festejar el cambio, elogiar y el uso de tareas (Neipp, s.f.).

Hipnosis ericksoniana. Milton H. Erickson creía que las personas tienen dentro de sí las capacidades naturales necesarias para superar dificultades, resolver problemas, entrar en trance y experimentar todos los fenómenos del trance, según lo describe O'Hanlon (2001).

De acuerdo a Haley (1991), la forma en que Erickson trabajaba con sus pacientes era la siguiente: a) Concepción del ser humano como una fuente de recursos y capacidades, la mayoría de ellos inconscientes. b) Tera- 
pia estratégica en el sentido de que Erickson asumía la responsabilidad de influir directamente en las personas. c) Aliento de la resistencia en el sentido de prescribir el "síntoma". d) Ofrecimiento de una alternativa peor o "ilusión de alternativas", en donde el usuario aparentemente decide sobre dos opciones que el terapeuta le propone, pero finalmente hace lo que el terapeuta planea. e) Provocar cambios a través del uso de metáforas. f) Empleo del espacio y la posición, es lo que comúnmente se le Ilama escenificación en la terapia estructural. g) Énfasis en lo positivo, magnificándolo y utilizándolo. h) Utilización de lo que el paciente ofrece, incluyendo el síntoma, la postura, el lenguaje, etc. i) Consideración de las etapas del ciclo vital en las metas terapéuticas. j) Evitación de la exploración del sí mismo. I) Uso de la amnesia y el control de la información.

Respecto al caso que se presenta en este trabajo, y como lo señalan Guy y Schnur (2004), y Mendoza y Capafons (2009), la hipnosis ha demostrado ser una herramienta efectiva para el trabajo clínico en problemas físicos y emocionales, lo que incluye la ansiedad.

Es así como en el presente estudio de caso se describe la intervención terapéutica en una familia cuyo motivo de consulta era eliminar la ansiedad en su hijo mayor, pero que durante el proceso terapéutico se logró abrir el conflicto que limitaba el funcionamiento familiar, apoyándonos en el enfoque centrado en soluciones y la hipnosis ericksoniana.

\section{MÉtodo}

\section{Identificación del caso}

\section{Participantes}

Se trató de un matrimonio de clase media, en ciclo vital pertinente de hijos adultos. La familia estaba compuesta por 4 integrantes: madre, de 47 años de edad, ama de casa; el padre de 48 años de edad y comerciante de autopartes; el hijo mayor varón de 20 años de edad, estudiante del tercer semestre de publicidad, además de que trabaja con su padre, y la hija menor de 14 años que estudiaba la secundaria al tiempo que cursaba una carrera técnica en comercio.

En cuanto a la estructura familiar, inicialmente se encontraron: límites rígidos, conflicto en el holón conyugal, la triangulación y centralidad negativa recaía en el hijo, conflicto en el holón fraternal, y la jerarquía la tenía el esposo.

\section{Análisis del motivo de la intervención}

La madre Ilamó a las instalaciones de INFASI para solicitar terapia debido a que su hijo mayor presentaba crisis de ansiedad, al grado de ponerse violento, y refirió que se preocupaba mucho por su padre cuando éste se encontraba fuera o bien no regresaba temprano a casa; así que solicitaba que se le ayudara a que su hijo supiera controlar esa ansiedad.

\section{Antecedentes del problema}

Entre los antecedentes del problema importantes a señalar, mencionados durante las sesiones, la madre refirió sentimientos de celos hacia el esposo cuando salía de casa y no sabía dónde se encontraba, incluso cuando estaba embarazada de su hija sentía que le hervía la sangre por esa situación y llegaba el momento en que salía a buscarlo, pero esta situación disminuyó hasta que nació su hijo. En ese tiempo ella padeció dermatitis atópica, así que, durante los primeros tres años de su hijo, el padre se había hecho cargo del cuidado del mismo.

Otra situación más que podría resultar importante para el caso fue que cuando el hijo tenía 6 años de edad, había presenciado un intento de secuestro que iba dirigido a su padre, hubo disparos de un arma de fuego, pero no había sido requerido el apoyo psicológico. Y, finalmente, la hija padecía alopecia.

\section{Descripción y análisis del problema}

Durante la segunda sesión, cuando los padres asistieron solos a terapia, la información obtenida resultó importante para el análisis del problema ya que unos días antes de esta sesión, el hijo se había alterado al grado de ponerse agresivo incluso decía que quería golpear.

Cuando le preguntaban por qué se ponía así, él solamente respondía que era el temor a que le pasara algo a su padre, culpando además a su madre porque ella era la responsable de cuidar que el esposo regresara temprano a casa. Por su parte, el esposo mencionó que no había presenciado las crisis de su hijo, sólo se lo habían platicado. Por otro lado, se comentó también que la hija sólo se refugiaba en la computadora cuando las crisis del hermano se presentaban; sin embargo, ella presentaba una sintomatología de alopecia.

Finalmente, otro dato relevante fue que el padre había notado que cuando el hijo tenía fiesta y salía, la pre- 
ocupación no se presentaba, aunque la madre reportaba que sí. También comentaron que el hijo estaba saliendo con una joven no muy del agrado de la madre, pero que no le impedía estar con ella ni hacer planes ya que había compartido con ambos padres su deseo de irse a Europa porque la novia se iría para allá.

Después de la segunda sesión se hizo la revisión del caso, encontrando seis hipótesis:

- Posiblemente cuando la madre se recuperó de la dermatitis atópica, el padre cedió en atención a su hijo y tal vez el hijo presentó crisis de ansiedad para obtener atención o cariño de éste.

- Posiblemente la esposa sentía celos y preocupación por su esposo, y al no saber cómo expresarlo, era probable que lo estuviera comunicando por medio de sus hijos, quizá con la creencia de poder controlar a su esposo.

- Probablemente el hijo no estaba de acuerdo sobre control que tenía la madre hacia el padre y una forma de comunicar su desacuerdo era por medio de las crisis de ansiedad y preocupación.

- Probablemente la periferia del padre se debía a que él no se dejaba controlar por su esposa, de esta manera se desencadenaba un conflicto conyugal.

- Posiblemente la alopecia de la hija era una forma de mostrar su desacuerdo del control que ejercía su madre en ella; aunado a esto, cabía la posibilidad de ser una manera de comunicar atención de los padres, ya que ellos estaban enfocados en la crisis de ansiedad de su hermano.

De estas hipótesis se generó una sistémica donde se presuponía que los celos y la preocupación que la esposa sentía hacía su marido los estaba comunicando por medio de sus hijos, tal vez de esta manera ella creía que podía tener algún tipo de control sobre él.

Sin embargo, era probable también que los hijos no estuvieran de acuerdo con ella y a su vez sus síntomas (crisis de ansiedad y preocupación por parte del hijo, y alopecia por parte de la hija) eran una prueba de ello. En cuanto al esposo, quizás él prolongaba sus salidas de la casa debido a los constantes reclamos que le hacían su esposa y sus hijos.

\section{Plan de intervención}

\section{Descripción del plan de intervención}

Tras la información obtenida en la primera sesión (familiar), segunda sesión (de pareja) y tercera sesión (individual, ya que sólo asistió la madre), se optó por trabajar con el autocontrol de la ansiedad de la madre a través del modelo de intervención centrado en las soluciones y la hipnosis clínica, bajo el supuesto de que la madre tiene los recursos y fortalezas necesarios para mover el cambio del sistema familiar completo.

\section{Establecimiento de los objetivos y las metas del plan de intervención}

Objetivo: Disminuir la ansiedad como problema en el funcionamiento familiar.

\section{Metas de intervención:}

a. Identificar el ciclo que en la familia llevaba la ansiedad como síntoma y como mantenimiento del funcionamiento familiar.

b. Evidenciar el problema familiar.

c. Romper ciclo del problema.

d. Establecer estrategias en la familia para alentar el cambio.

\section{Técnicas de evaluación del plan de intervención}

\section{Instrumentos}

Se utilizó el esquema de intervención de la Maestría en Hipnosis y Terapia Familiar Breve Sistémica para la Ilamada telefónica (recuperar principalmente el motivo de consulta y formar un familiograma que permitiera generar algunas hipótesis), primera sesión (dar un encuadre al modelo de trabajo, identificar recursos positivos y lados fuertes de los clientes; establecer la meta y objetivos terapéuticos), y el seguimiento de las mismas (revisión de tareas, evaluación de objetivos).

Todas las sesiones fueron videograbadas con la finalidad de dar seguimiento y poder evaluar a la familia por medio de su discurso verbal tomando en cuenta su lenguaje analógico (Beyebach, 2006).

\section{Procedimiento del trabajo terapéutico}

Se realizó una primera llamada al cliente para recabar la información general del caso (familiograma y motivo de consulta), y concretar la fecha de la primera sesión.

Ya en la primera sesión, a los asistentes a terapia se les explicaron las condiciones en las que se llevaría a cabo la terapia, siguiendo el procedimiento de la Maestría en Hipnosis y Terapia Familiar Breve Sistémica. Se mencionó que sería un trabajo de supervisión en vivo, donde estarían presentes la familia, el terapeuta, un equipo terapéutico y un supervisor del caso. Las sesiones programadas fueron 5 (correspondientes al calendario de la maestría), la duración de cada una de ellas 
fue de aproximadamente una hora, con un espacio de 15 días.

\section{Aplicación del plan de intervención}

\section{Desarrollo de las sesiones}

\section{Primera sesión:}

Asistió la familia completa, y cuando se estaba leyendo el reglamento de usuarios y autorización para grabar la sesión, el hijo mayor comenzó a mover la cabeza negativamente, mencionando que no quería el servicio terapéutico con tanta gente, entonces el equipo terapéutico y supervisora le comentaron a la familia que se dieran la oportunidad de conocer el tipo de servicio que se ofrecía, si no era de su agrado que no los tomaran. Aceptaron entonces quedarse a una plática informativa del ciclo vital de la familia.

El mensaje final fue que, si como familia decidían tomar el proceso terapéutico, no sólo nos bridan la oportunidad de trabajar en la situación que les preocupaba a ellos sino que también se daban la oportunidad de lograr cambios como familia. Al terminar la sesión, el padre mencionó que continuarían con el proceso terapéutico. Además, se pudo obtener como meta terapéutica que el hijo mayor supiera controlar su ansiedad y preocupación por su padre. Suponíamos en esta sesión que lo que podría ayudar en esta familia era recuperar sus logros y antecedentes positivos que ellos mismos se reconocen como ser una familia unida.

\section{Segunda sesión:}

Ambos padres llegaron puntuales a la sesión, refirieron que de la plática informativa de la sesión anterior habían reflexionado sobre su etapa como familia y entonces sus hijos habían decidido acudir a terapia de forma individual. Al continuar indagando sobre antecedentes del problema, la madre hizo referencia a que ella había estado presente cuando su hijo había tenido crisis de ansiedad, la cual era desencadenada cuando el padre llegaba tarde a casa, o no se reportaba si es que tenía que salir. Mencionó que ella, por su parte, comenzaba a orar y es así como notaba que el hijo se tranquilizaba.

Ambos padres ratificaron su motivo de estar en terapia para que su hijo controlara su ansiedad, y en esta sesión se les dejó claro que sí se les podría ayudar, pero como familia tendrían que hacer cambios, como por ejemplo, cuando el hijo presentara alguna crisis de ansiedad, la madre no debía estar presente (incluso debía salirse de casa). Se les mencionó también que posiblemente también tuvieran que dejarlo ir a Europa, aunque fuera por perseguir a la novia, así tal vez regresara con otra persona, pero independiente.

Como mensaje final se les comentó lo siguiente: "una familia se mueve como un sistema, lo que sucede con alguno de sus integrantes está muy relacionado con todo lo demás. En algunos momentos el hijo no se preocupa por su padre, el que quiera irse a Europa con la novia, eso quiere decir que es posible controlar sus crisis porque no siempre las tiene. Por otro lado, ustedes son una familia sabia, que saben tomar decisiones, ya tienen velatorio y tienen un lugar preparado que de cierta forma da certeza, y tranquilidad y seguridad; y por cosas como éstas están aquí como familia que dejará que su hijo adulto tome decisiones y sea autosuficiente, así como su hija adolescente empieza a tomar sus decisiones. En todas las familias hay problemas, pero con inteligencia se resuelven. Lo que nos preguntamos ahora como terapeutas es, ustedes como padres de familia qué están dispuestos a hacer para que su familia esté mejor, porque con lo que nos han comentado y la experiencia que tenemos en el trabajo clínico, creemos que su caso no es fácil pero los podemos ayudar, es más, posiblemente tengan que romper un paradigma familiar, empezando quizá por la lealtad a situaciones que no es posible seguir manteniendo de la misma forma, de la misma forma volteen hacia sus hijos, lo que les va a costar trabajo es desprenderse de sus hijos, la independencia de sus hijos no es fácil.

Su hijo quiere controlar, es válido, es necesario, el control sobre él deriva en la capacidad de independencia. Se va ir con una mujer, sí y cómo lo va hacer, no ponerle trabas y tal vez regrese con otras habilidades, se deben unir como pareja, que retomen sus salidas, el actúa como niño, qué es lo que lo limita a estudiar, hay escuela pública, si él quiere hacer algo, que lo dejen, la señora ya no haga nada por su hijo, y que no le diga nada, les va a costar trabajo, porque la señora le dice que hacer y él no le hace caso, entonces para ella es un desgaste emocional, porque ella está acomodando...Su hijo no está loco, tiene mucha energía y una manera de sacarla es preocupándose por su padre, cuando tenga otra crisis de ansiedad, sólo sálgase, déjelo a él solo".

\section{Tercera sesión A:}

No asisten a la sesión a pesar de que la señora llamó por teléfono a la terapeuta para comentarle que asistiría su hijo. 


\section{Tercera sesión B (un mes después de la segunda se-} sión):

La señora llegó sola y mencionó que su esposo no quiso asistir. Refirió sentirse liberada de un miedo crónico, mismo que le había ayudado a controlar su preocupación. Ella comentó que el hecho de que su esposo e hijo no hubieran asistido a sesión era una forma de no hacerse responsables. También dijo que las crisis de ansiedad de su hijo ya no habían aparecido; sin embargo, su hija se había salido de casa sin permiso en dos ocasiones, la primera a una colonia cerca de su casa para irse a desayunar después de salir de la iglesia y la segunda ocasión para encontrarse con su novio, aunque los padres del novio la regresaron a su casa.

En ambas ocasiones el padre se había puesto violento con su hija, así que se le cuestionó a la madre qué hacia ella mientras observaba a su esposo en esas condiciones, y ella respondió que nada, aun cuando no estaba de acuerdo en la forma de actuar. Así que se le comentó que, si bien no era la forma más adecuada de actuar de ambos padres para que la situación se calmara y no volviera a suceder, se debería de enfocar en buscar y accionar con respeto. La energía que tienen para pelearse como esposos la tendrán que ocupar para ponerse de acuerdo, y de manera firme tendría que decirle a su esposo que no apoyaba ni estaba de acuerdo por la violencia que ejerce sobre sus hijos. Se preguntó también qué hacían como padres para superar que su hija tenía más fortaleza que su hijo mayor, y qué para que la hija obedeciera o fuera capaz de enfrentar al mundo, en ambos casos se necesitaba templanza y paciencia de ella como madre.

Finalmente, se le dijo que ella era catalizadora de todas las emociones, comprendía a su esposo, a sus hijos, pero parecía que a ella nadie la comprendía, tenía que estar tranquila para responder de forma asertiva; por tanto, se le aplicó una técnica de hipnosis "Un lugar de serenidad" (de Corydon, que consiste en llevar a la persona a un lugar especial, tranquilo y seguro, centrando su atención en su respiración, en como su cuerpo se relaja y en como su cuerpo recarga baterías de sentimientos gratificantes, de tal manera que estas sugestiones permanezcan en ella y las practique en el día) con la finalidad de que la señora centrará su atención en sus emociones, en su respiración, y en consecuencia, canalizarlas de manera favorable, y se le dejó de tarea practicarla todos los días en casa, porque su familia se le va a desmoronar si sigue pensando que ella puede, advirtiendo también que lo sucedido en la sesión de hoy no lo platicara con su familia.

\section{Cuarta sesión:}

Se trabajó de manera individual con la señora. Se habló sobre la realización de la tarea (ejercicio de hipnosis) y de los beneficios obtenidos al darse cuenta que estaba más tranquila, de encontrase consigo misma, logró concentrar su atención en las oraciones matutinas que realiza, y todo ello había ayudado a que su angustia disminuyera. Comentó que de presentarse un acontecimiento como el ocurrido con su hija hace un mes, ella tomaría el tiempo para calmarse, pensar y actuar acertadamente. Refirió que las discusiones con su esposo habían disminuido, también percibía un ambiente familiar más tranquilo, dándose cuenta que si quería un cambio tenía que empezar por ella misma como el bajar su tono de vOz cuando se percibe gritando.

Entonces se le preguntó que si no tuviera estos problemas qué pasaría, qué estaría haciendo, cuál sería la diferencia de estar relajada a estar a la expectativa, a lo que ella respondió que entendía que si quería ver a su hijo manejando la ansiedad tenía que verse ella sin ansiedad. También se cuestionó y dejó como mensaje el que pensara en lo que le tocaba hacer para que su hijo no copiara su forma de actuar y de sentir, y respondía que entonces debería estar cero alterada, tono de voz bajo, incluso eso había comenzado a notar en la última vez que su hijo tuvo crisis de ansiedad, ya que ella guardó silencio y pudo pensar.

Finalmente, se trabajó con ella la técnica de hipnosis "Orquesta de sanación" (de Roberts Dilts; que consiste en una breve inducción que guía a la persona a un estado de relajación, cuyo foco de atención es en una parte del cuerpo, que sienta o se considere sano de forma que dirija la atención en cómo se extiende por todo el cuerpo, y este sentimiento podrá evocarlo posteriormente de tal manera que la sanación se expande favorable y paulatinamente, es así como se obtiene ganancias a la sanación), para continuar reforzando en canalizar de manera funcional sus emociones y que además ella ya estaba experimentando sentirse tranquila, y se le dejó de tarea que este ejercicio de relajación lo practicara todos los días además de no mencionar nada de lo sucedido en la sesión en casa, esto porque ella tendría que saber describir cómo es estar tranquila, de esta manera no sólo podría actuar de manera acertada, sino que también podrá apoyar y ayudar a su hijo si se presentará otra crisis de ansiedad. En este sentido ella era quién pondría ejemplo de cómo tranquilizarse para que las enfermedades somáticas de su familia disminuyeran paulatinamente. 


\section{Quinta sesión:}

De acuerdo con el calendario escolar, esta era la última sesión programada, tal como se había especificado a la familia desde la primera vez que asistieron. Así que consideró trabajarla en tres momentos: revisión de tareas, trabajo con hipnosis y cierre de la terapia. Cabe mencionar que, dados los resultados reportados, no fue necesario el uso de técnicas de hipnosis. Se describe a continuación el trabajo realizado.

La señora asistió puntualmente a la cita y comentó haber realizado en un promedio de 8 ocasiones el ejercicio de relajación, dándose cuenta de que mientras más relajada se sentía la actitud hacia su esposo y hacia sus hijos en momentos de conflicto cambiaba favorablemente. Y de eso se había percatado más en una ocasión en que discutió con su hija acerca de la responsabilidad, honestidad y de que no respetaba los horarios, y su esposo había intervenido a su favor para pedirle a su hija que respetara y escuchara a su madre y no tenía porque levantar el tono de voz, en señal de respeto, cuando solicitaba algo.

En cuanto a su hijo, había presentado nuevamente una crisis de ansiedad, la había llamado del cine y sólo para preguntarle si su padre ya estaba en casa, él había pedido que ella se comunicara con el padre para saber dónde estaba, y ella le respondió que no haría tal cosa y le colgó el teléfono, en la segunda llamada trató de calmar a su hijo, él le refirió que su padre ya había apagado el celular; en la tercera Ilamada escuchó a su hijo más tranquilo, y comentaba que no había seguido el juego, con esto ella se sentía tranquila y así actuó y confió, recordando lo que se había trabajado ya en la terapia. Incluso después de esa ocasión, sus hijos ya habían comentado sobre su cambio, que la veían más tranquila y más calmada.

Algo que le preocupaba en ese momento era no tenerle confianza a su hija, incluso ya había platicado con ella acerca de la sexualidad y los embarazos no deseados. Le molestaba que no le respondiera la llamada telefónica; sin embargo, ya había reconocido que era un círculo lo que sucedía: "te cacho en la mentira - no te creo - no te dejo salir - necesito salir/conocer, pero mi madre no me deja salir (negociar) - ella no me cree, por lo tanto, miento para poder salir". Se le preguntó cómo podría ella romper el círculo ya que el sistema familiar mantenía su atención en las actividades que sus hijos hacían y que de alguna manera estaban fuera de lugar, por lo tanto el hecho de cambiar o romper esto alteraría favorablemente el patrón del sistema familiar que tanto le preocupaba y que además parecía ser una constante en la forma de comunicación, todo el sistema se movía a través de situaciones como éstas y, aunque se le dejó como tarea el pensar en esta solución, también se le comentó que la edad de su hija era complicada, y al no darle permiso a su hija, ésta no crecerá ni aprenderá así que tendría que empezar a soltar, la preocupación no la llevaría a ningún lado por lo tanto tenía que ocuparse por medio del ejercicio de relajación en encontrar un punto de equilibrio en una negociación donde ella como madre sí pudiera darle permiso de acuerdo a las costumbres de la familia y a la edad de su hija.

Se anotó también que era un buen momento para trabajar esto sola donde pusiera en práctica los recursos que la tranquilidad le ha traído, con mente más clara se puede pensar más claro y mejor, evitando llegar a que su hija agravara su alopecia o ella o cualquiera de la familia desarrollará una enfermedad somática. Para finalizar la sesión, se acordó con la señora continuar el proceso terapéutico, aunque fuera del programa académico, así que se programaron dos sesiones más a las que la señora ya no asistió. En la llamada de seguimiento mencionó que seguía tranquila, había aprendido a controlar su ansiedad y estaba aún trabajando en su cambio personal.

\section{Presentación de resultados}

En la tabla 1 se muestra en resumen el trabajo realizado por sesión, las intervenciones terapéuticas realizadas, así como los objetivos programados y la evaluación de los mismos. En cuanto a los resultados alcanzados, en la tabla se puede observar el avance que se tuvo en el trabajo de la disminución de ansiedad y ante su relación con la funcionalidad familiar.

\section{Análisis de los cambios que produjo el plan de intervención}

A partir de la descripción del caso, y como ya hacía mención Yalóm (como se citó en De Castro \& García, 2011), la ansiedad puede surgir al darse cuenta de que nuestros valores y sistemas de creencia se destruyen, y quizá nos sintamos fracasados en algún proyecto, por mínimo que sea, porque la incertidumbre viene acompañando la idea de que no todo cambio es positivo. En esta familia, la etapa del ciclo vital (con hijos adolescentes) representaba también una constante para que la independencia se permitiera, pero la manifestación de la ansiedad resaltaba más como el problema a considerar.

Con lo señalado, resultó interesante el proceso terapéutico en sí mismo, ya que la madre como cliente había solicitado apoyo para su hijo (paciente); sin em- 


\begin{tabular}{|c|c|c|c|}
\hline Sesión & INTERVENCIONES TERAPÉUTICAS & Objetivos Terapéuticos & Avances Reales \\
\hline 1 & $\begin{array}{l}\text {-Complementariedad } \\
\text {-Consejo especializado }\end{array}$ & - Concretar meta terapéutica. & $\begin{array}{l}\text { - La familia decidió quedarse a la plática informativa, y } \\
\text { se mantuvo atenta a lo que se decía. } \\
\text {-Se definió meta terapéutica. } \\
\text {-Se reconocieron como una familia unida. }\end{array}$ \\
\hline II & $\begin{array}{l}\text { - Repetición del mensaje } \\
\text { - Orientaciones para la negociación } \\
\text { - Conversación para el cambio } \\
\text {-Preguntas presuposicionales } \\
\text {-Búsqueda de excepciones. }\end{array}$ & $\begin{array}{l}\text { - Identificar el ciclo que en la familia } \\
\text { llevaba la ansiedad como síntoma y como } \\
\text { mantenimiento del funcionamiento familiar. } \\
\text { - Ampliar la excepción del problema. }\end{array}$ & $\begin{array}{l}\text { - Reflexionaron sobre su etapa como familia. } \\
\text { - Los hijos decidieron asistir a terapia de forma } \\
\text { individual. } \\
\text {-Ambos padres se confrontaron sobre las excepciones } \\
\text { en que el problema no se presentaba. }\end{array}$ \\
\hline III & $\begin{array}{l}\text {-Preguntas presuposicionales } \\
\text {-Pregunta milagro } \\
\text {-Excepciones } \\
\text { - Co-crear realidades } \\
\text { - Atribución de control } \\
\text { - Técnica de hipnosis "Un lugar de } \\
\text { serenidad" } \\
\text {-Tarea }\end{array}$ & $\begin{array}{l}\text {-Evidenciar el ciclo del problema. } \\
\text { - Romper con ciclo del problema. } \\
\text {-Establecer estrategias de cambio. }\end{array}$ & $\begin{array}{l}\text { - La madre se dio cuenta que se liberó de un miedo } \\
\text { crónico y controló su preocupación. } \\
\text {-Hijo no presentó crisis de ansiedad. } \\
\text { - En la técnica de hipnosis, se logró un trance profundo } \\
\text { lo que llevó a la señora a relajarse y comenzar la } \\
\text { necesidad de buscar opciones para mantenerse en ese } \\
\text { estado. }\end{array}$ \\
\hline IV & $\begin{array}{l}\text {-Preguntas presuposicionales } \\
\text { - Co-crear realidades } \\
\text {-Anclar las soluciones } \\
\text { - Festejar el cambio } \\
\text { - Técnica de hipnosis "Orquesta de } \\
\text { sanación" } \\
\text { - Tarea } \\
\text {-Elogiar el cambio }\end{array}$ & $\begin{array}{l}\text {-Evidenciar avances. } \\
\text {-Mantener estrategias de cambio. }\end{array}$ & $\begin{array}{l}\text { - La madre realizó la tarea de ejercicios de relajación, } \\
\text { y logró darse cuenta que estaba más tranquila, } \\
\text { encontrándose consigo misma, logró concentrar su } \\
\text { atención en sus oraciones y todo ello ayudó a que la } \\
\text { angustia disminuyera } \\
\text { - La madre mencionó que se tomaría el tiempo para } \\
\text { calmarse, para pensar y así actuaría acertadamente } \\
\text { - Las discusiones entre los cónyuges disminuyeron, } \\
\text { percibiéndose un ambiente familiar más tranquilo en } \\
\text { su casa. } \\
\text { - Se dio cuenta que el cambio debía empezar por ella, } \\
\text { como bajar su tono de voz y que la percibieran sin } \\
\text { ansiedad. }\end{array}$ \\
\hline v & $\begin{array}{l}\text {-Elogiar. } \\
\text {-Atribuir el control. } \\
\text {-Promover activamente la cooperación } \\
\text { con la familia. }\end{array}$ & $\begin{array}{l}\text {-Mantener estrategias de cambio. } \\
\text {-Reforzar recursos y fortalezas personales } \\
\text { que influyen en la funcionalidad familiar. }\end{array}$ & $\begin{array}{l}\text { - La señora continuó realizando el ejercicio de relajación } \\
\text { esto a su vez se dio cuenta que sentía una actitud de } \\
\text { cambio favorable de su esposo e hijos. } \\
\text {-El esposo validó a la esposa frente a los hijos. } \\
\text { - La familia percibe a la señora más tranquila y más } \\
\text { calmada. }\end{array}$ \\
\hline
\end{tabular}

bargo, el transcurso de las sesiones fue definiendo al paciente identificado: en la primera sesión se trabajó con la familia completa, en la segunda sesión asistieron ambos padres únicamente y a partir de la tercera sesión asistió sólo la madre, quedando ella como paciente identificado. Y es que como lo mencionó Charles (2008), ante los conflictos encubiertos por la pareja, lo primero que llega a terapia es trabajar con su rol de padres para un problema prioritario de un hijo, ya que la terapia de pareja será en una segunda o tercera fase del trabajo; es decir, pudiera ser que la conducta de los niños y adolescentes de alguna manera es una metáfora de la conducta de algunos de los padres; o de la relación de los padres.
Será hasta la segunda etapa en la que se pueda entrar a los problemas de comunicación y conflictos de relación conyugal. Además, a partir del trabajo realizado en la segunda sesión, y de acuerdo a la información que proporcionaron los padres, se pudieron generar hipótesis que sirvieron de eje base para el proceso terapéutico (que era desaparecer las crisis de ansiedad en el hijo); sin embargo, también éstas se fueron modificando de acuerdo al avance de las sesiones logrando cumplir el objetivo terapéutico de disminuir la ansiedad del hijo, sólo cuando la madre se dio cuenta que podía autocontrolar la ansiedad y como resultado (y paulatinamente) desaparecerla, canalizando sus emociones de forma 
asertiva y por tanto el funcionamiento familiar también tenía cambios.

En el momento en que a ambos padres se les mencionó que sí se les podría ayudar (primera sesión), pero tendrían que hacer cambios dentro del sistema familiar para lograr control de la ansiedad de su hijo, hubo un cambio importante, ya que también habría repercusiones en la independencia de sus hijos. Como padres deberían respetar las decisiones que tomaran sus hijos, aunque ellos no estuvieran de acuerdo, de esta manera también se fortalecería la relación de pareja. Y esto sucedió porque la esposa de alguna manera insistía en que sus hijos se preocupaban tanto por su padre, al grado de que ella notaba como reprimían sus emociones, y así ella parecía forzar a que su esposo se diera cuenta del daño; sin embargo, asumimos la hipótesis de que dicha preocupación era de ella, de su sistema de creencias y valores, y no de los hijos.

Específicamente sobre las sesiones se identificaron los siguientes resultados: En la primera sesión, como un

"La ansiedad se
convierte en un ciclo
de comunicación y en
un estilo de vida"
consejo especializado, el tema central fue el ciclo vital de la familia; en este sentido se logró alianza terapéutica con la familia donde sólo se habló de las diferentes etapas y responsabilidades de cada holón individual, es así como la familia pudo darse cuenta que las interacciones y dinámica de la misma deben encaminarse a la funcionalidad.

Para la segunda sesión, donde asistieron sólo los padres, el proceso terapéutico se enfocó indagando sobre las premisas de la terapia centrada en soluciones; la idea de que posiblemente los celos y preocupación por el esposo, al no saber cómo expresarlo, es probable que lo esté comunicando por medio de sus hijos, tal vez de esta manera ella cree que puede controlar a su esposo. En este sentido, los esposos se dieron cuenta que deberían ponerse de acuerdo y retomar su relación de pareja, con la intención de que la comunicación entre ambos era vital para el funcionamiento familiar de acuerdo a la etapa en la que estaba viviendo entre cambios e incertidumbres.

En la tercera sesión, la hipnosis ericksoniana fue de gran apoyo ya que se normalizó una situación problemática que parecía de deserción del esposo a la terapia, y se brindó una oportunidad para que la madre-esposa-mujer aprendiera y reaprendiera a tomar decisiones de manera tranquila en su familia, si ella lograba eso, la familia cambiaría también. Ante este trabajo, los cambios reportados por la señora sobre el miedo controlado y la tranquilidad que se siente al soltar lo que no le pertenece se hicieron evidentes.

Para la cuarta sesión, los recursos personales y la fortaleza de la señora se volvían visibles para todos, ya que podía concentrar su atención en sus oraciones, mencionó sentirse más tranquila y darse cuenta que durante el ejercicio de hipnosis podía estar consigo misma, refirió también que los cambios que estaba logrando le ayudarían en la toma de decisiones acertadas como pensar y actuar de manera tranquila ante las crisis de ansiedad del hijo y las de ella misma.

En la quinta sesión la señora reportó cambios claros, dándose cuenta de que su cuerpo estaba tranquilo porque se sabía más calmada, más relajada, más confiada, hacia conciencia antes de actuar y prueba de ello fue cuando su hijo tuvo una crisis de ansiedad y ella le colgó el teléfono rompiendo con el patrón de elevar el nivel de ansiedad, en este sentido ella no se conectaba con la ansiedad de él, de esta manera ella se daba cuenta que su hijo también estaba logrando dismi-

nuir su ansiedad y preocupación por su padre.

Finalmente, si bien el funcionamiento familiar podría tener más cambios hablando terapéuticamente, en la llamada de seguimiento, la señora reportaba el mantenerse tranquila y su familia también tomando ya decisiones con sus hijos adolescentes. Lo cual implicó que, con las intervenciones trabajadas, se logró poner a flote los recursos personales y familiares.

\section{Conclusiones}

En este estudio de caso sobre la ansiedad y su relación con el funcionamiento familiar, se manifiesta la idea de que las personas ya disponen de habilidades y recursos que pueden usar para resolver sus problemas, y que con herramientas como intervenciones desde el modelo centrado en soluciones y de hipnosis clínica, el trabajo individual, que a su vez gira en torno a un cambio pequeño, sólo se moviliza a los clientes a encontrar o buscar sus propias soluciones de manera más efectiva.

En el trabajo se destacó pensar que la ansiedad es parte de la cotidianidad, siempre que sea en nivel moderado y que permita ser funcional en las actividades al individuo y a la familia; sin embargo, en ocasiones parece indicar que ésta fue aprendida y que funciona como un ciclo en la comunicación, es más, puede ser ya un estilo de vida y que llega un momento en que no a todo el sistema le conviene mantenerse en este patrón y busca 
salirse, provocando incluso quiebre en otros miembros del sistema y por lo tanto un cambio.

En el caso, la familia se había percatado que la ansiedad del hijo no estaba siendo canalizada de la mejor manera posible, al grado de causar incomodidad sobre todo en la interacción familiar, es así como decidieron acudir a terapia psicológica, pero enfocando la atención en el hijo mayor; sin embargo, el trabajo terapéutico se centró en la madre y en las interacciones familiares, cuya característica fundamental fue el enfoque que se le dio a las conversaciones donde el contenido varió dependiendo de los acontecimientos de la paciente, aunado a ello se fueron ampliando los cambios que reportó, y que en muchas ocasiones no se había percatado que sucedieron, por eso es que hubo la necesidad de enfatizar a que continuara realizando aquello que la hacía sentir bien.

Resultan interesantes los cambios que fueron reportados por la señora, ya que al inicio del proceso la familia rechazó el servicio terapéutico, en este sentido ella se dio la oportunidad de crecer para su bienestar, sin duda alguna que asistió puntualmente a las sesiones pensando en beneficio de su familia, los cambios que reportó fueron su herramienta para comunicarle a su familia que era posible controlar y reducir la ansiedad.

Cabe mencionar que el modelo centrado en soluciones y la hipnosis ericksoniana, usados como estrategias de intervención, dieron la pauta para negociar un problema resoluble y en este sentido, los pacientes se tranquilizan ya que tienen la certeza que abordarán sus problemas de una manera eficiente fortalecidos en sus recursos y potencialidades personales. Es por ello entonces que se recomienda ampliamente ambas formas de trabajo, por su nobleza en profundizar en cualidades e ideas positivas de solución que trae el paciente a consulta.

\section{Referencias}

Alchaer, J. R.; Bahsas, F.; Hernández, N. \& Salinas, P. J. (1994). Relación entre el funcionalismo familiar, el estrés y la ansiedad. Med-ULA, Revista de la Facultad de Medicina, Universidad de Los Andes, 3(3-4), 81-88. Recuperado de erevistas.saber.ula.ve/index.php/medula/article/ view/5611

Beyebach, M. (2006). 24 ideas para una psicoterapia breve. Barcelona: Herder.

Beyebach, M. (2013). La terapia familiar breve centrada en soluciones. Manuscrito del 2013, no publicado. El texto aparecerá en (Alicia Moreno, ed.), Modelos de Terapia Familiar. Universidad Pontificia de Comillas. Recuperado de http://psyciencia.psyciencia.netdna-cdn.com/wpcontent/uploads/2013/12/El-modelo-de-la-TerapiaFamiliar-Centrada-en-Soluciones-.pdf
Charles, R. (2008). Terapia breve sistémica en soluciones para parejas y padres. Esquemas, guías y ejemplos para una práctica exitosa. México: CREE-SER.

De Castro, A. \& García, G. (2011). Psicología Clínica. Fundamentos Existenciales. Ediciones Uninorte.

Fuentes, M.L. (16 de julio de 2013). Salud mental: una agenda invisible. Periódico Excélsior. Recuperado de http://www. excelsior.com.mx/nacional/2013/07/16/909049

Goldman, H. (2001). Psiquiatría general. México: Manual Moderno.

Guy, Montgomery. \& Schnur, J. (2004). Eficacia y aplicación de la hipnosis clínica. Papeles del Psicólogo, 89. Recuperado de http://www.papelesdelpsicologo.es/vernumero. asp?id=1180

Haley, J. (1991). Terapia no convencional. Buenos Aires: Amorrortu.

Isaacs, A. (2000). Enfermería de salud mental y psiquiátrica (2a ed.). Madrid: McGraw-Hill/Interamericana de España.

Méndez, L.; Giraldo, O.; Aguirre-Acevedo, D. \& Lopera, F. (2010). Relación entre ansiedad, depresión, estrés y sobrecarga en cuidadores familiares de personas con demencia tipo alzheimer por mutación e280a en presenilina 1. Revista Chilena de Neuropsicología, 5(2), 137-145. Recuperado de www.redalyc.org/articulo.oa?id=179314915007

Mendoza, M. E. \& Capafons, A. (2009). Eficacia de la hipnosis clínica: resumen de su evidencia empírica. Papeles del psicólogo, 30(2), 98-116. Recuperado de http://dialnet. unirioja.es/servlet/articulo?codigo $=3002275$

Moreno, A. (2013).). Modelos de Terapia Familiar. Manuscrito no publicado. El texto aparecerá en Alicia Moreno (Ed.). Universidad Pontificia de Comillas. Recuperado de http:// psyciencia.psyciencia.netdna-cdn.com/wp-content/ uploads/2013/12/El-modelo-de-la-Terapia-FamiliarCentrada-en-Soluciones-.pdf

Neipp, M. C. (s.f.). Introducción a la terapia breve centrada en soluciones. Presentación. Recuperada de http://umh1365. edu.umh.es/wp-content/uploads/sites/95/2013/02/ TCSFinal2013.pdf

O'Hanlon, B. (2001). Desarrollar Posibilidades, un itinerario por la obra de uno de los fundadores de la terapia breve. España: Paidós.

O ' Hanlon, W. \& Weiner-Davis, M. (2010). En busca de soluciones, un nuevo enfoque de psicoterapia. España: Paidós.

Quintana, A. \& Sotil, A. (2014). Influencia del clima familiar y estrés del padre de familia en la salud mental de los niños. Revista de Investigación en Psicología, Norteamérica, 3(2). Recuperado de <http://revistasinvestigacion.unmsm.edu. pe/index.php/psico/article/view/4990/4054>

Rosário, P; Núñez, J. C.; Salgado, A.; González-Pineda, J. A.; Valle, A; Joly, C. \& Bernardo, A. (2008). Ansiedad ante los exámenes: relación con variables personales y familiares. Psicothema, 20(4), 563-570. Recuperado de http://www. psicothema.com/pdf/3523.pdf

Salazar, Y.; Veytia, M.; Márquez, O. \& Huitrón, G. (2013). Relación entre satisfacción con el ambiente familiar y depresión en adolescentes. Psicología y Salud, 23(1), 141 148. Recuperado de http://revistas.uv.mx/index.php/ psicysalud/article/view/524

Soria, R.; Ávila, E. \& Rodríguez, N. (2014). Efectos del trabajo 
académico en la salud y en las relaciones familiares de hombres y mujeres estudiantes universitarios. Revista Electrónica de Psicología Iztacala, 17(1), 150-164. Recuperado de http://www.iztacala.unam.mx/carreras/ psicologia/psiclin/vol17num1/Vol17No1Art8.pdf
Zavala, M. R.; Ríos, M. C.; García, G. \& Rodríguez, C. P. (2009). Funcionalidad familiar y ansiedad en pacientes adultos con enfermedad crónica. Chía, Colombia, 9(3), 257-270. Recuperado de www.redalyc.org/pdf/741/74112147006. pdf 


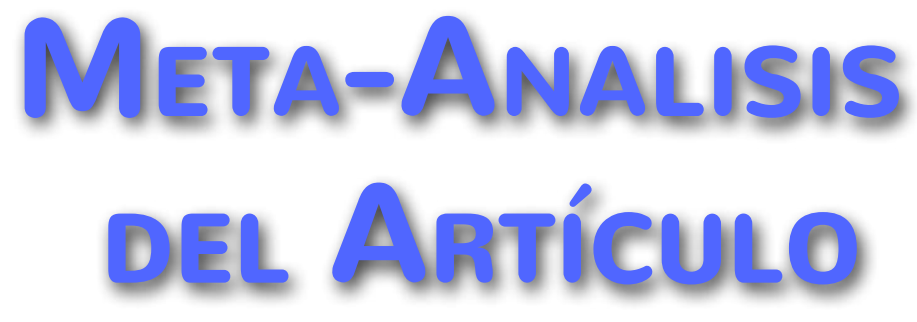




\section{Dimensión Cuantitativa}

\section{Perfil de Evaluación entre pares}
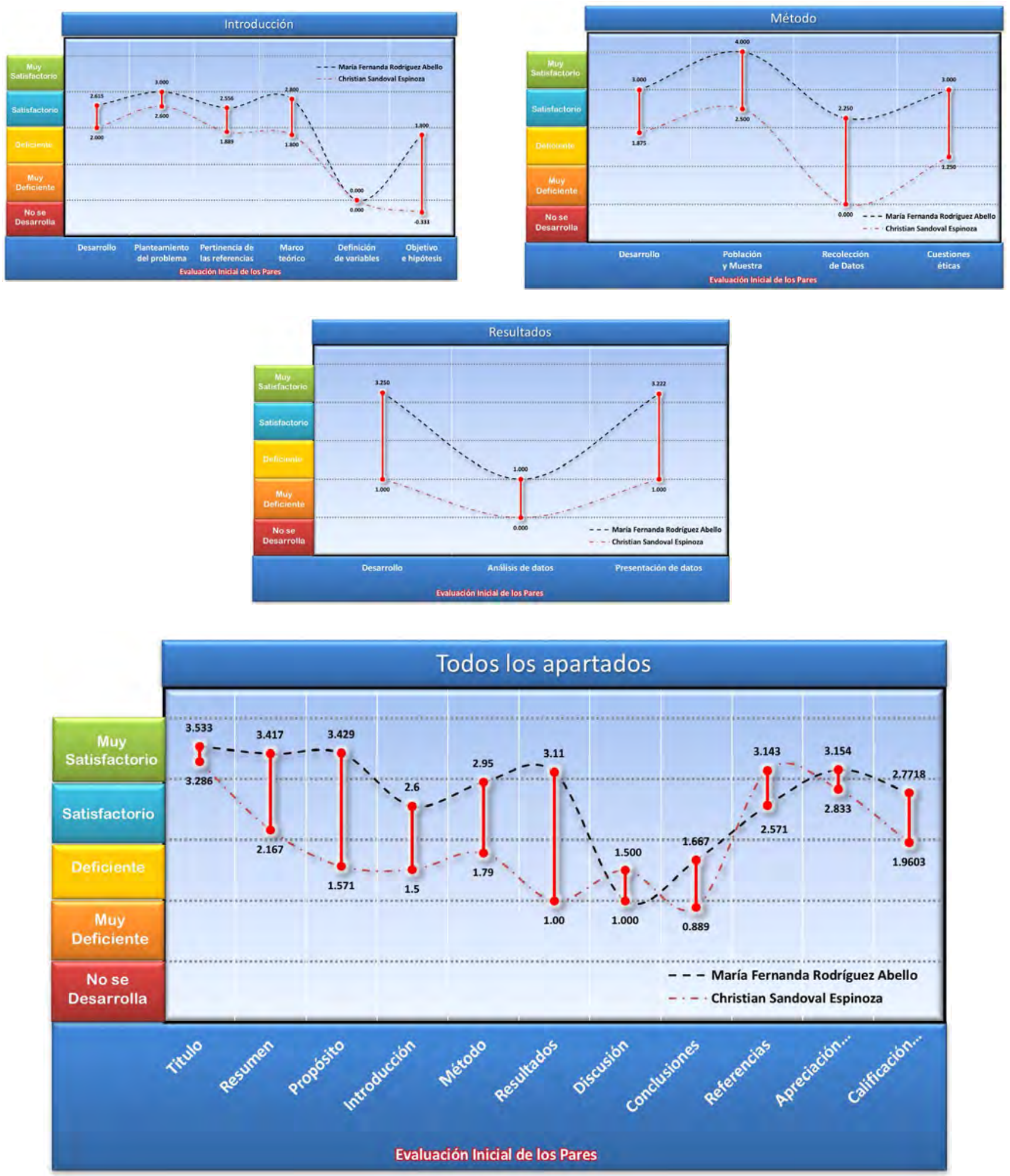

$\cos \rightarrow \infty x$ 


\section{Índice de Concordancia}

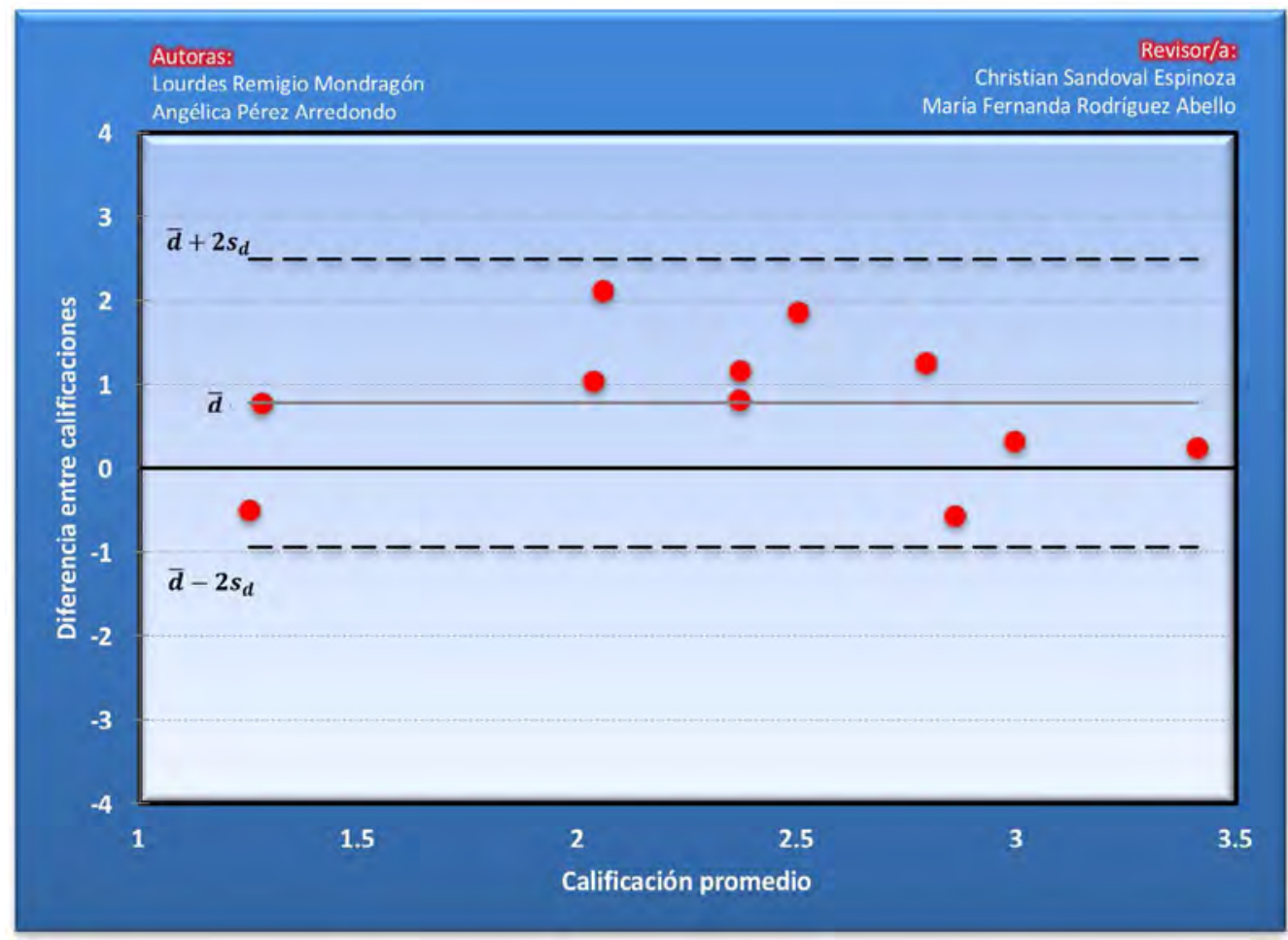

\section{Índice de Acuerdo}

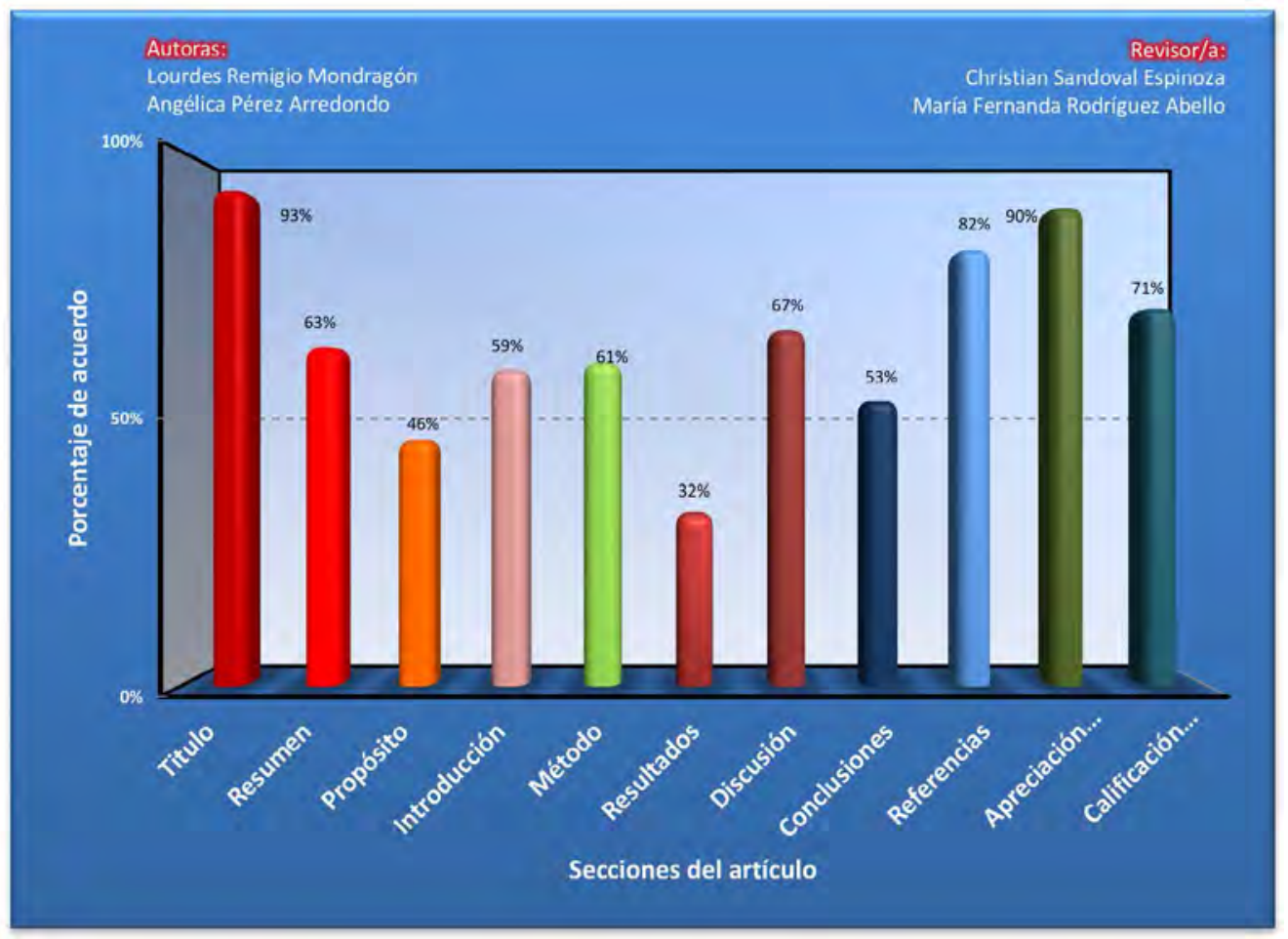

\section{WHAिक्म}




\begin{tabular}{|c|c|}
\hline REVISOR 1 & REVISOR 2 \\
\hline Christian Sandoval Espinosa & María Fernanda Rodríguez Abello \\
\hline \multicolumn{2}{|c|}{ Título/Autoría } \\
\hline $\begin{array}{l}\text { No posee el formato que marca el APA respecto a la } \\
\text { presentación del artículo }\end{array}$ & $\begin{array}{l}\text { El artículo tiene como objetivo revisar el impacto } \\
\text { de la terapia sobre los niveles de ansiedad que ex- } \\
\text { perimenta una familia, por lo que el título no es el } \\
\text { coherente, no van a revisar la relación entre funcio- } \\
\text { namiento familiar y ansiedad }\end{array}$ \\
\hline
\end{tabular}

\section{Resumen}

Es necesario revisar la redacción general del resu-

El resumen contiene muchos problemas de mecanografía y/u ortografía men y reemplazar palabras como mamá y papá por madre y padre. La traducción a inglés es muy deficiente

\section{Próposito del Estudio}

El propósito carece de rigurosidad y congruencia con el método y sobre todo con el tipo de hipótesis planteadas
Definen varios propósitos en el artículo ya que según el título se va a revisar la relación entre funcionamiento familiar y ansiedad, en el resumen el objetivo es ver de qué manera la terapia ayuda a disminuir los niveles de ansiedad en una familia y en el marco teórico dicen qu la hipótesis de la que parten es que la ansiedad es heredada

\section{INTRODUCCIÓN}

En el marco teórico no se trabaja el modelo de hipnosis eriksoniana, el cual se empleó en casi el 50\% del caso y el tipo de hipótesis son epitemológicamente incompatibles con la visión sistémica, dado que son lineales y no recursivas o circulare
Revisar el uso de las normas APA para las citas textuales. Cuando se usan siglas como APGAR la primera vez debe escribirse el nombre completo y luego entre paréntesis la sigla. Mencionar de otra manera "los del estrato socioeconómico laboral obrero, los obesos". Revisar la redacción general, sobre todo en el título "intervención terapéutica" esa sección está desconectada del artículo ya que habla de la terapia por desde diferentes enfoques, habría que enfocarla a soluciones e hipnosis y conectarla con el tema de ansiedad, además de buscar investigaciones al respecto 


\section{MÉTOdo}

El mismo artículo posee rubros adicionales que no fueron desarrollados, se ha referencia a técnicas que nunca se explican o describen, y se cometió una falta de ética importante, se dio de "alta" el caso porque terminó el semestre, lo cual denota poco interés por el cliente y su proceso, lo que consideraron fue el tiempo cronológico
Revisar la redacción, utilizar iniciales hace la lectura complicada, es mejor usar seudónimos o nombres falsos y hacer la aclaración de que los nombres fueron cambiados. Se explica sesión a sesión lo que sucede con la familia, es importante hace énfasis, más que en la información que se obtuvo (eso va en los resultados) en la manera en que se intervino, cuales fueron los métodos utilizados, aunque para definir esto es importante definir primero la orientación del artículo, cual es el objetivo del mismo. Menciona que las sesiones fueron videograbadas lo que es un dato importante para la recolección de información, pero no menciona si este fue el instrumento que se utilizó o fue otro y luego que procedimiento se utilizó para organizar la información (se transcribieron las sesiones, se tomaron notas de ellas...)

\section{Resultados}

Se introdujo mucho la subjetividad de la terapeuta, no se citaron los resultados obtenidos a partir de las preguntas de escala y los demás miembros de la familia (esposo y hijos) tampoco reportaron mejoría, dado como resultado conclusiones dudosas. Además, no se recuperan las hipótesis como parte del cierre
Teóricamente hablando es importante tener cuidado con varias cosas: hay varias hipótesis enunciadas las cuales están desconectadas una de la otra, según mencionan en el artículo que fueron las que se elaboraron la primera sesión y para efectos del artículo es mejor mencionar cual de ellas fue la que orientó el caso o elaborar una hipótesis sistémica que conecte todos los puntos; la tabla que presentan resulta útil para resumir cada sesión, sin embargo hay cosas importantes para señalar: en objetivos de la sesión 1 aparecen "lados fuertes" lo cual es una técnica, no puede ser el objetivo utilizar una técnica en esa sesión, mas bien la técnica tiene un objetivo, la misma situación se presenta para preguntas presuposicionales, pregunta del milagro, por lo que es necesario revisarlo. Lo interesante para presentar como resultado podría ser presentar el efecto que tuvo el uso de cierta técnica, de cierta intervención en los consultantes, por lo que habría que encontrar la conexión, pero, insisto, es necesario antes definir cual es el objetivo del artículo. Revisar APA para la presentación de figuras y tablas 


\section{Dimensión Cualitativa}

\section{Discusión}

La discusión es muy breve, tanto que no da pie para la reflexión o un meta discurso basado en lo reportado en cada cédula de sesión
No hay un análisis de resultados ni una relación de estos con la teoría o con otros artículos escritos referentes al tema

\section{Conclusiones}

Las conclusiones están disgregadas del marco teórico y el desarrollo o método.
Es importante mencionar posibles campos de investigación futuras en las conclusiones y también tener cuidado con el uso de palabras como "comprobar" ya que en este caso resultan antiparadigmáticasdos

\section{RefERENCIAS}

Agregar mas referencias de no más de 8 años de antigüedad.
Es importante fortalecer más la cantidad y actualizar los documentos revisados. Es importante atender los criterios APA. 


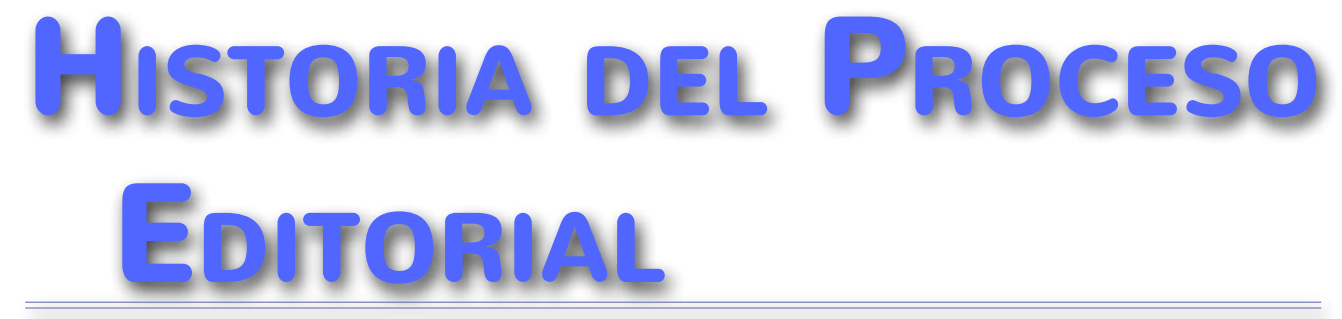

